\title{
The prevalence of Epstein-Barr Virus-positive lymphoid cells in nasal mucosa: an extremely rare event*
}

Seung Yeon $\mathrm{Ha}^{1}$ and Sanghui Park²

Department of Pathology, Gachon University Gil Medical Center, Incheon, Korea

2 Department of Pathology, and Global Top 5 Program, Ewha Womans University School of Medicine, Seoul, Korea
Rhinology 52: 403-405, 2014

DOl:10.4193/Rhino13.178

*Received for publication:

October 20, 2013

Accepted: May 12, 2014

\begin{abstract}
Background: The prevalence of EBV (Epstein-Barr virus)-positive lymphoid cells is unknown. Because EBV is implicated in the etiology of extranodal NK/T-cell lymphoma, nasal type (nasal ENKL), the presence of EBV-positive lymphoid cells (EPLs) in nasal mucosa specimens is expected. This study evaluated the presence of EBV-positive lymphoid cells in the nasal mucosa of 420 patients who had undergone surgical resection of lesions of the nasal cavity due to nasal septal deviation, chronic paranasal rhinosinusitis, chronic hypertrophic rhinosinusitis, nasal polyps, allergic rhinitis, papillomas, and cysts.
\end{abstract}

Methodology: Three representative 1.0-mm-diameter core biopsies were taken from one paraffin-embedded donor tissue block per case and subsequently arranged in new recipient paraffin blocks with a trephine. EBV in situ hybridization study was performed to detect EPLs.

Results: None of the cases demonstrated EPLs.

Conclusion: The presence of EPLs in the nasal mucosa is an extremely rare event in immunocompetent individuals. Therefore, the detection of EPLs in nasal biopsy specimens should prompt the pathologist to perform further testing to exclude the possibility of nasal ENKL.

Key words: Epstein-Barr virus, nasal mucosa

\section{Introduction}

Epstein-Barr virus (EBV) is implicated in the etiology of nasopharyngeal carcinoma (NPC) and extranodal NK/T-cell lymphoma, nasal type (nasal ENKL). Although nasal ENKL may also involve the nasopharynx, where most NPCs arise, it usually occurs in the anterior nasal cavity ${ }^{(1)}$. Although EBV-positive lymphocytes (EPLs) have been found in normal nasopharyngeal mucosa and adenoids ${ }^{(2-4)}$, the prevalence of EBV infection in non-neoplastic lymphoid cells of the nasal mucosa in immunocompetent, lowrisk individuals has not been studied well. Considering that nasal ENKL is almost always EBV-positive and occurs mostly in the nasal cavity ${ }^{(5)}$, the presence of EPLs in nasal mucosa specimens is expected. We were interested in determining the prevalence of EPLs in the nasal mucosa of low-risk populations. Therefore, we used an EBV in situ hybridization technique to determine the presence of EPLs in the nasal mucosa tissues of 420 patients who had undergone surgical resection of lesions of the nasal cavity due to nasal septal deviation, chronic paranasal rhinosinusitis, chronic hypertrophic rhinosinusitis, nasal polyps, allergic rhinitis, papillomas, and cysts.

\section{Materials and methods}

\section{Study population}

We reviewed the H\&E slides and clinical and pathologic reports of 420 patients who had undergone surgical resection for nasal septal deviation, chronic paranasal rhinosinusitis, chronic hypertrophic rhinosinusitis, nasal polyps, allergic rhinitis, papillomas, and cysts between January 1999 and October 2004 at the Gil Medical Center. The source of mucosal tissue in patients with septoplasty was the anterior portion of inferior concha. Mucosa 
was not taken from the septum. Clinicopathologic parameters, such as age, gender, histologic diagnosis, and type of operation, were evaluated.

\section{Tissue microarray method}

Three representative 1.0-mm-diameter core biopsies were taken from one paraffin-embedded donor tissue block per case and subsequently arranged in new recipient paraffin blocks with a trephine (Quick-Ray, UNITMA). Serial sections from the tissue microarray blocks were used in the EBV in situ hybridization. An adequate case was defined as that in which the nasal mucosa occupied all three cores.

\section{EBV in situ hybridization}

In situ hybridization for EBV-encoded RNA (EBER) was performed on the Bond Max Immunohistochemical Stainer ${ }^{\circledR}$ (Leica Microsystems), using fluorescein-conjugated oligonucleotide probes (EBER Probe, Leica Biosystems, Newcastle, UK) according to the following protocol. First formalin-fixed, paraffin-embedded tissue sections were deparaffinized, and the slides were incubated with enzyme for 15 minutes, after which the fluorescein-conjugated EBER probe was placed on the slides and incubated for 1 hour at $37^{\circ} \mathrm{C}$. After incubation with the EBER probe, the tissue sections were incubated with a mouse anti-fluorescein antibody for 15 minutes, post-primary antibody for 8 minutes, and polymer for 8 minutes, followed by colorimetric development with DAB. We used EBV-positive diffuse large B-cell lymphoma as a positive control (Figure 1).

\section{Results}

Clinical characteristics
This study included 420 patients who had undergone surgical resection for nasal septal deviation $(n=182)$, chronic paranasal rhinosinusitis ( $n=85$ ), chronic hypertrophic rhinosinusitis ( $n=$ 77 ), nasal polyps ( $n=47)$, allergic rhinitis $(n=20)$, papillomas ( $=5)$, and cysts $(n=4)$. Surgical operations included endoscopic sinus surgery $(n=45)$, conchotomy $(n=98)$, polypectomy $(n=$ 23), Caldwell operation ( $n=4)$, septoplasty $(n=41)$, submucosal resection ( $n=11)$, and unspecified $(n=198)$. The mean age was 31.6 years (range, 6 to 80 years), and most patients were male ( $\mathrm{n}$ $=302,71.9 \%)$.

\section{EBV in situ hybridization}

The results of the in situ hybridization analysis failed to demonstrate EPL in any of the 420 cases (Figure 2).

\section{Discussion}

This study evaluated the presence of EPLs in the nasal mucosa of 420 immunocompetent individuals by constructing tissue microarray (TMA) blocks. No EPLs were found in the nasal mucosal tissues of any patient. Although EPLs are known to occur in the nasopharynx, adenoids, and lymph nodes ${ }^{(2-4,6,7)}$, the presence of EPLs in the nasal mucosa has not been evaluated thoroughly. Tao et al. reported EPLs in 11 out of 13 nasal polyps in Chinese patients ${ }^{(8)}$. However, Kozak et al. failed to observe EPLs in Canadian patients ${ }^{(9)}$. The increased frequency of EBV infection in the nasal polyps of Chinese patients suggests that the natural history of EBV infection may vary according to environmental and/or genetic factors ${ }^{(8)}$. Our study included 47 cases of nasal polyps, but none of them showed EPLs. Numerous etiologies for nasal polyps have been proposed, with allergic and infectious



Figure 1. Representative scanning image of tissue microarray slide in patients with diffuse large B-cell lymphoma (EBV in situ hybridization). The tumor cells were diffusely positive for EBV in one case (Inset x200).

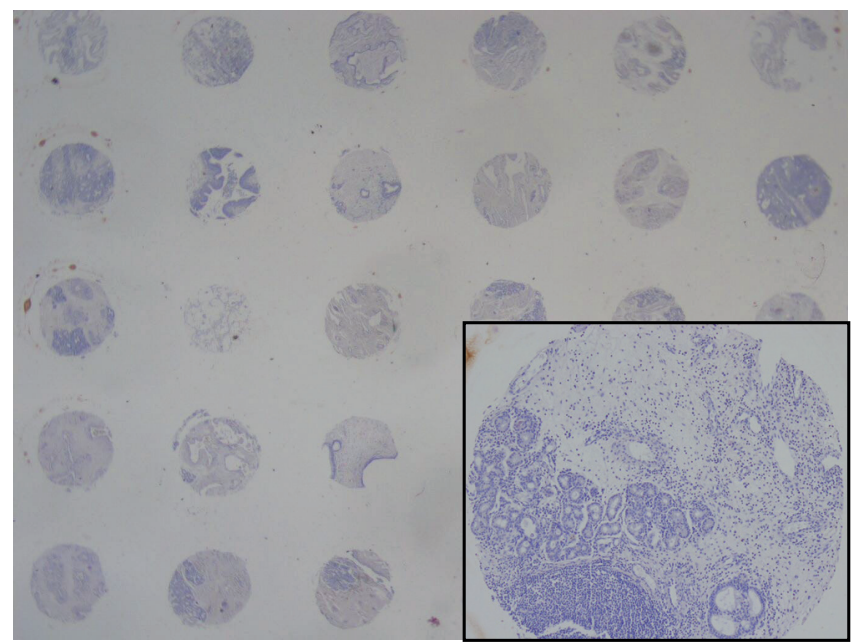

Figure 2. Representative scanning image of tissue microarray slide in immunocompetent populations with nasal mucosa (EBV in situ hybridization). The lymphoid cells were negative for EBV (EBV in situ hybridization) (Inset x200). 
causes predominant ${ }^{(10-12)}$. Weill considered a viral etiology for nasal polyps nearly 63 years ago ${ }^{(13)}$. However, viral culture of nasal polyp tissue demonstrated no viruses in one study ${ }^{(14)}$. In addition to nasal polyps, we also investigated the presence of EPLs in a variety of nasal lesions, such as nasal septal deviation, chronic paranasal rhinosinusitis, chronic hypertrophic rhinosinusitis, allergic rhinitis, papillomas, and cysts, but no EPLs were identified.

Our study has some limitations. We made TMA blocks to perform EBV in situ hybridization on as many cases as possible. Although three cores were taken per case, this was probably not sufficient to represent whole tissues. A previous study found that EPLs were few in number and tended to be scattered rather than forming distinct foci in post-nasal space biopsy specimens ${ }^{(15)}$, and our previous study also demonstrated a few scattered EPLs in nasopharynx biopsy specimens ${ }^{(16)}$. Although these studies were performed using post-nasal space biopsy specimens or nasopharynx biopsy specimens, we can infer from the results that EPLs in the nasal mucosa, if present, may be widely distributed rather than clumped in aggregates. Further studies using whole blocks of nasal mucosa specimens will be needed to demonstrate the presence of EPLs.

Despite these limitations, the present study highlights an important point: the presence of EPLs in the nasal mucosa is a very rare event in immunocompetent individuals. In the nasopharynx, EPLs can be found occasionally and are mainly B-cells.
Therefore, the presence of EPLs in the nasopharynx does not necessarily indicate nasal ENKL. In contrast, even if a few scattered EPLs are identified in nasal mucosa biopsy specimens, thorough evaluation should be performed, regardless of whether the EPLS are T- , NK- or B-cells, to exclude the possibility of nasal ENKL. Because there are currently no diagnostic criteria for nasal ENKL that define the cut-off numbers of EBV-infected NK/T cells, the possibility of so-called 'in situ' or indolent nasal ENKL has not been proposed yet as a provisional disease entity in cases in which small numbers of NK/T cell are EBV-positive ${ }^{(16)}$.

In conclusion, the presence of EPLs in the nasal mucosa is a very rare event in immunocompetent individuals. Therefore, further evaluation is necessary when EPLs are detected in nasal biopsy specimens.

\section{Acknowledgement}

None

\section{Authorship contribution}

SYH reviewed slides, constructed tissue microarray blocks and wrote this manuscript. SP reviewed slides and wrote this manuscript.

\section{Conflicts of Interest}

The authors declare that they have no conflicts of interest.

\section{References}

1. Ho FC, Choy D, Loke SL, et al. Polymorphic reticulosis and conventional lymphomas of the nose and upper aerodigestive tract: a clinicopathologic study of 70 cases, and immunophenotypic studies of 16 cases. Hum Pathol. 1990; 21: 1041-1050.

2. Endo LH, Vassallo J, Sakano E, Brousset P. Detection of Epstein-Barr virus and subsets of lymphoid cells in adenoid tissue of children under 2 years of age. Int J Pediatr Otorhinolaryngol. 2002; 66: 223-226.

3. Tao Q, Srivastava G, Chan AC, Chung LP, Loke SL, Ho FC. Evidence for lytic infection by Epstein-Barr virus in mucosal lymphocytes instead of nasopharyngeal epithelial cells in normal individuals. J Med Virol. 1995; 45: 71-77.

4. Tao Q, Srivastava G, Chan AC, Ho FC Epstein-Barr-virus-infected nasopharyngeal intraepithelial lymphocytes. Lancet. 1995; 345: 1309-1310.

5. Steven H. Swerdlow EC, Nancy Lee Harris, Elaine S. Jaffe, Stefano A. Pireli, Harald Stein, Jurgen Thiele, James W. Vardiman. WHO Classification of Tumours of Haematopoietic and Lymphoid Tissues. Lyon: International Agency for Research on Cancer (IARC), 2008.

6. Niedobitek G, Herbst H, Young LS, et al Patterns of Epstein-Barr virus infection in non-neoplastic lymphoid tissue. Blood. 1992; 79: 2520-2526.

7. Tonoyama $Y$, Teramoto $N$, Sarker $A B$, et al. Detection of Epstein-Barr virus RNA and related antigens in non-neoplastic lymphoid lesions. Acta Med Okayama. 1996; 50: 89-96.

8. Tao Q, Srivastava G, Dickens P, Ho FC. Detection of Epstein-Barr virus-infected mucosal lymphocytes in nasal polyps. Am J Pathol. 1996; 149: 1111-1118.

9. Kozak FK, Mahony JB, Chernesky MA, et al. Nasal polyposis: in search of a viral etiology using DNA hybridization. J Otolaryngol. 1991; 20: 404-407.

10. Drake-Lee AB, Lowe D, Swanston A, Grace A. Clinical profile and recurrence of nasal polyps. J Laryngol Otol. 1984; 98: 783-793.

11. Drake-Lee AB, McLaughlan P. Clinical symptoms, free histamine and IgE in patients with nasal polyposis. Int Arch Allergy Appl Immunol. 1982; 69: 268-271

12. Jones E, Frenkiel S, Small P, Rochon L. Immunopathological characteristics of nasal polyps. J Otolaryngol. 1987; 16: 19-22.

13. Weille FL. The effect of nasal and sinus surgery upon the manifestations of allergy. $\mathrm{N}$ Engl J Med. 1950; 242: 43-48.

14. Dunnette SL, Hall MM, Washington JA, 2nd, et al. Microbiologic analyses of nasal polyp tissue. J Allergy Clin Immunol. 1986; 78:
102-108.

15. Lees JF, Goodeve AC, Arrand JE, Ghosh AK, Jones PH, Arrand JR. Detection of EBV DNA in post-nasal space biopsy tissue from asymptomatic EBV-seropositive individuals. J Med Virol. 1992; 37: 30-38.

16. Ha SY, An J, Park S. Detection of EpsteinBarr virus-infected lymphoid cells in nasal mucosa or nasopharynx: appearances can be deceptive. Virchows Arch. 2013; 462: 391-397.

Sanghui Park, MD, PhD

Assistant Professor

Department of Pathology

Ewha Womans University School of

Medicine

911-1 Mok-dong, Yangcheon-gu,

Seoul, 158-710,

Republic of Korea

Tel: +82-2-2650-5731

Fax: +82-2-2650-2879

E-mail: spark0430@ewha.ac.kr 